\title{
BX-1: A New Detector Window for Microanalysis Applications
}

\author{
Jonathan Abbott ${ }^{1}$, Jared Sommer ${ }^{1}$, Brian Law ${ }^{1}$, Josh Wong ${ }^{1 *}$ \\ 1. Moxtek, Orem, UT \\ * Corresponding author: jwong@moxtek.com
}

A new x-ray transmission window, BX-1, with improved low energy transmission and high temperature tolerance has been developed. The BX-1 window uses a low atomic number inorganic thin film which is thinner than polymer-based membranes. This allows for better X-ray transmission and fewer atomic absorption edges. The inorganic film also exhibits better gas barrier properties compared to polymerbased membranes. Additionally, The BX-1 window has high temperature tolerance for improvements in detector manufacturing. A non-epoxy adhesive material offers high temperature performance up to $200^{\circ} \mathrm{C}$ with $1.2 \mathrm{~atm}$ differential pressure. The new window is shown to be resistant to corrosion from humidity and temperature. The materials are all high-vacuum compatible with very low outgassing rates. The window has very good mechanical strength despite using a thinner membrane than current x-ray windows.

An X-ray window is an indispensable component for the X-ray detector. The window isolates the detector's vacuum environment from the sample's variable pressure environment, which in some applications can be at atmosphere, while allowing transmission of x-rays from the sample to the detector. The window can also prevent contamination of the detector from the sample environment. In addition to holding the pressure difference between sample and detector without breaking, the window has to be gas-impermeable, charge-dissipating, stable over time (with exposure to many environmental variables) and reject visible light. The materials used for the X-ray window should be comprised of low atomic number elements to maximize the $\mathrm{x}$-ray transmission through the window. A major challenge for energy-dispersive x-ray detector equipment is to increase the signal capture throughput per unit time. Doing this allows for faster analysis with lower signal to noise ratio and less potential beam damage to the sample with the same amount of signal generated from the sample. An x-ray detector window that has higher x-ray transmission will allow for faster analysis, all other factors being equal.

Figure 1 compares the modelled x-ray transmission of the new BX-1 window to AP3. Table 1 outlines the mechanical performance of the BX-1 window. Long-term helium permeability of BX-1 compared to AP3 is shown in Figure 2. 


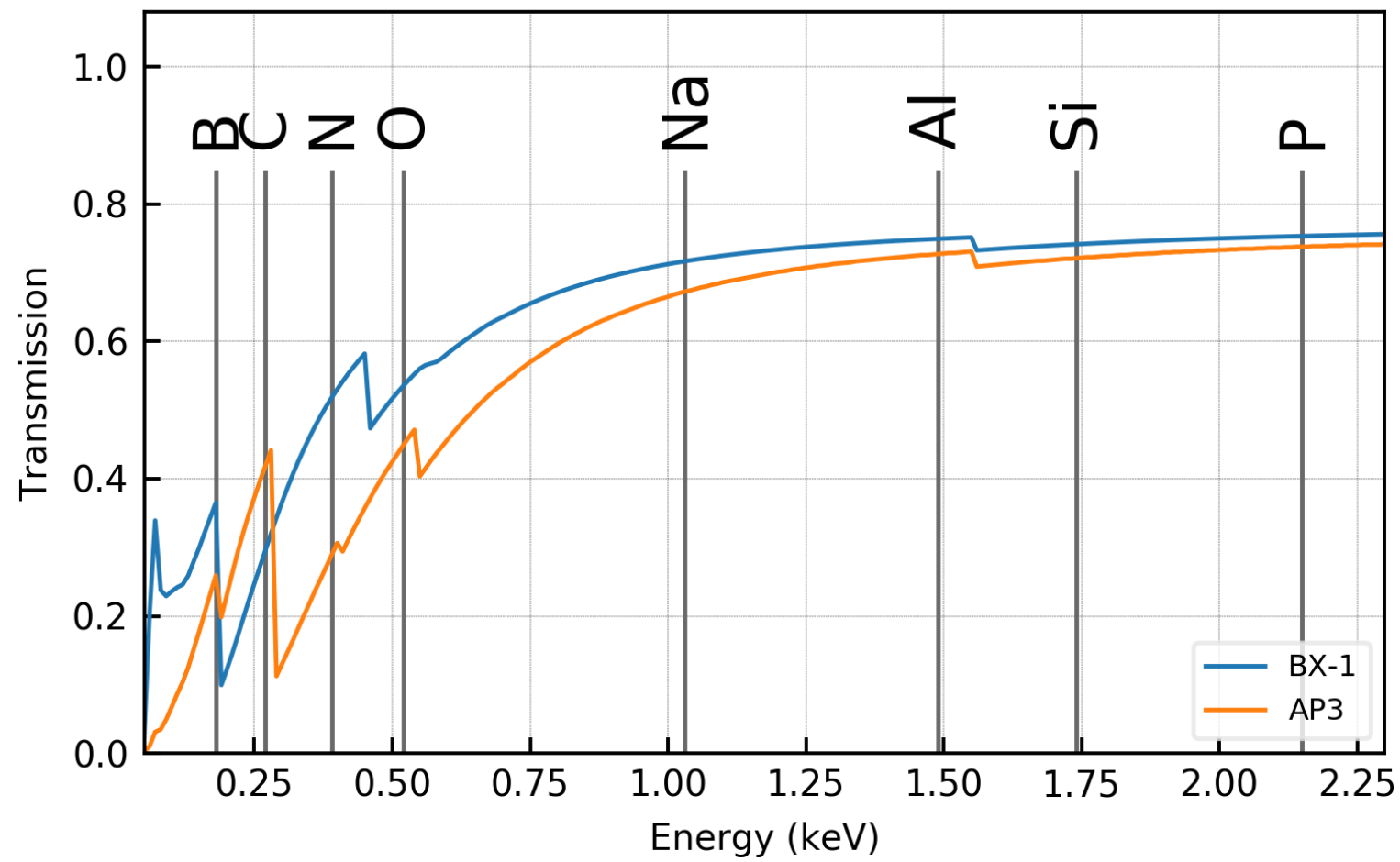

Figure 1. X-Ray transmission comparison between BX-1 and AP3

Table 1. Mechanical testing summary

\begin{tabular}{|l|l|}
\hline Mechanical Property & Value \\
\hline Forward Pressure & $3.5 \mathrm{~atm}$ \\
\hline Back Pressure & $1.8 \mathrm{~atm}$ \\
\hline
\end{tabular}

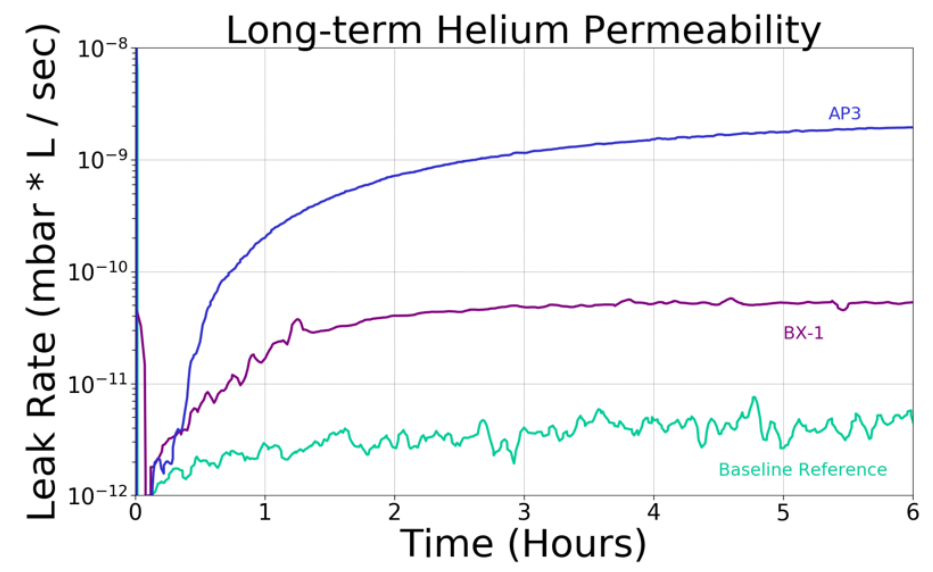

Figure 2. Visible light transmission for BX-1 